Journal of Applied AnALysis

Vol. 7, No. 2 (2001), pp. 243-255

\title{
COMPOSITIONS OF SIERPIŃSKI-ZYGMUND FUNCTIONS AND RELATED COMBINATORIAL CARDINALS
}

\author{
F. JORDAN \\ Received November 27, 2000 and, in revised form, April 14, 2001
}

\begin{abstract}
A cardinal related to compositions of Sierpiński-Zygmund functions will be considered. A combinatorial characterization of the cardinal is given and is used to answer some questions of K. Ciesielski and T. Natkaniec. It is shown that the bounding number of the continuum may be strictly smaller than continuum.
\end{abstract}

\section{Introduction}

Recall that $f \in \mathbb{R}^{\mathbb{R}}$ is called a Sierpiński-Zygmund function provided that the restriction $\left.f\right|_{X}$ is not continuous for any set $X \subseteq \mathbb{R}$ of cardinality $\mathfrak{c}$. We will denote the family of Sierpiński-Zygmund functions by SZ. We will be interested in resolving some problems in [3] about a cardinal, $\mathrm{C}_{\text {out }}(\mathrm{SZ})$, related to compositions of Sierpiński-Zygmund functions. We give a combinatorial characterization of $\mathrm{C}_{\text {out }}(\mathrm{SZ})$ and show that it has a close relationship to the higher cardinal generalization of the bounding number $\mathfrak{b}$ of $\omega$. Of particular

2000 Mathematics Subject Classification. Primary 26A15; Secondary 54A25.

Key words and phrases. Sierpiński-Zygmund functions, cardinal functions, singular cardinals.

ISSN 1425-6908（C) Heldermann Verlag. 
interest to us will be the value of the generalized bounding number when a cardinal is singular.

\section{Preliminaries}

In what follows we will use the following terminology and notation. Any notation not specifically defined can be found in [2]. Functions will be identified with their graphs. The set of all functions from a set $X$ into a set $Y$ will be denoted by $Y^{X}$. Given sets $X, Y, W$ and $f \in W^{Y}$ and $g \in Y^{X}$ we denote their composition by $f \circ g$.

The symbol $|X|$ will denote the cardinality of the set $X$. The successor of a cardinal $\kappa$ will be denoted by $\kappa^{+}$. We denote by $[X]^{<\kappa},[X]^{\kappa}$, and $[X]^{\leq \kappa}$ the sets of all subsets of $X$ of cardinality less than $\kappa$, equal to $\kappa$, and less than or equal to $\kappa$, respectively.

The cardinality of the real numbers $\mathbb{R}$ will be denoted by $\mathfrak{c}$. Given a cardinal number $\kappa$ we let $\operatorname{cf}(\kappa)$ denote the cofinality of $\kappa$. We say a cardinal $\kappa$ is regular provided that $\operatorname{cf}(\kappa)=\kappa$, otherwise we say $\kappa$ is singular. For functions $f, g \in Y^{X}$ let $[f=g]$ denote the set $\{x \in X: f(x)=g(x)\}$. We define $[f<g]$ and $[f \leq g]$ in a similar way when $\leq$ and $<$ are defined for $Y$.

We also will consider the following cardinals related to a cardinal $\kappa$.

$$
\begin{aligned}
& d_{\kappa}=\min \left\{|F|: F \subseteq \kappa^{\kappa} \&\left(\forall g \in \kappa^{\kappa}\right)(\exists f \in F)(|[f=g]|=\kappa)\right\} . \\
& \mathfrak{b}_{\kappa}=\min \left\{|F|: F \subseteq \kappa^{\kappa} \&\left(\forall g \in \kappa^{\kappa}\right)(\exists f \in F)(|[f \geq g]|=\kappa\} .\right.
\end{aligned}
$$

Note that $\mathfrak{b}_{\kappa} \leq d_{\kappa}\left[5\right.$, Proposition 1.3(2)]. When $\kappa=\omega$ the numbers $\mathfrak{b}_{\omega}$ and $d_{\omega}$ are equal to the bounding and eventually different numbers, respectively, both of which have been heavily studied, e.g. [1]. Notice that $\mathfrak{b}_{\kappa}$ is a regular cardinal [5, Proposition 1.3(1)] and that $\kappa<\mathfrak{b}_{\kappa}$ when $\kappa$ is regular.

For a cardinal $\kappa$ we let $\mathcal{S}_{\kappa}=\left([\kappa]^{<\kappa}\right)^{\kappa}$. Let $X$ and $Y$ be sets and define $\mathcal{R}(X, Y)=\left\{f \in Y^{X}:\left|f^{-1}(y)\right|<|X|\right.$ for every $\left.y \in Y\right\}$.

We will consider the following combinatorial cardinals which turn out to all be equal although it is not obvious that they are:

$\left(\lambda_{\kappa}^{1}\right) \lambda_{\kappa}^{1}$ is the smallest cardinality of an $F \subseteq \mathcal{S}_{\kappa}$ such that for any $g \in \kappa^{\kappa}$ there is an $f \in F$ such that $|\bigcup\{f(\xi): g(\xi) \in f(\xi)\}|=\kappa$,

$\left(\lambda_{\kappa}^{2}\right) \lambda_{\kappa}^{2}$ is the smallest cardinality of an $F \subseteq \mathcal{R}(\kappa, \kappa)$, such that there is a $g \in \kappa^{\kappa}$ such that for any $h \in \kappa^{\kappa}$ there is an $f \in F$ such that $|[h \circ f=g]|=\kappa$,

$\left(\lambda_{\kappa}^{3}\right) \lambda_{\kappa}^{3}$ is the smallest cardinality of an $F \subseteq \mathcal{R}(\kappa, \kappa)$, such that there is a $G \in\left[\kappa^{\kappa}\right]^{\kappa}$ such that for any $h \in \kappa^{\kappa}$ there is a $g \in G$ and an $f \in F$ such that we have $|[h \circ f=g]|=\kappa$

$\left(\lambda_{\kappa}^{4}\right) \lambda_{\kappa}^{4}$ is the smallest cardinality of an $F \subseteq \kappa^{\kappa}$ such that for every $g \in \kappa^{\kappa}$ there is an $f \in F$ such that $f[[g \leq f]]$ is unbounded in $\kappa$. 


\section{Results}

In $[3$, Theorem 4.9] it is shown that $f \in \mathcal{R}(\mathbb{R}, \mathbb{R})$ if and only if there is an $h \in \mathbb{R}^{\mathbb{R}}$ such that $h \circ f \in \mathrm{SZ}$.

The following cardinal is defined in [3]

$$
\left.\mathrm{C}_{\text {out }}(\mathrm{SZ})=\min \left\{|F|: F \subseteq \mathcal{R}(\mathbb{R}, \mathbb{R}) \&\left(\forall h \in \mathbb{R}^{\mathbb{R}}\right)(\exists f \in F)(h \circ f \notin \mathrm{SZ})\right\}\right\} .
$$

The following two propositions are established in [3, Theorems 4.11 and 4.14]:

Proposition 1. If $\mathfrak{c}$ is a regular cardinal, then $\mathfrak{c}<\mathrm{C}_{\text {out }}(\mathrm{SZ}) \leq 2^{\mathfrak{c}}$. Generally, $\operatorname{cf}(\mathfrak{c}) \leq \mathrm{C}_{\text {out }}(\mathrm{SZ}) \leq \mathfrak{c}^{\mathrm{cf}(\mathfrak{c})}$.

Proposition 2. If $\mathfrak{c}=\kappa^{+}$for some $\kappa$, then $\mathrm{C}_{\text {out }}(\mathrm{SZ})=d_{\mathfrak{c}}$.

The two propositions above suggest the following two problems about the cardinal $\mathrm{C}_{\text {out }}(\mathrm{SZ})$ which are posed in [3].

Problem 1. Is the assumption that $\mathfrak{c}$ is regular important in Proposition 1 ?

Problem 2. Can Proposition 2 be proved for any value of $\mathfrak{c}$ ? What if $\mathfrak{c}$ is regular?

We first give a combinatorial characterization of $\mathrm{C}_{\text {out }}(\mathrm{SZ})$ which is a corollary of the two following general theorems.

Theorem 3. If $\kappa \geq \omega$ is a cardinal, then

$$
\operatorname{cf}(\kappa) \leq \lambda_{\kappa}^{4}=\lambda_{\kappa}^{1}=\lambda_{\kappa}^{2}=\lambda_{\kappa}^{3} .
$$

Theorem 4. If $\kappa$ is singular, then $\operatorname{cf}(\kappa) \leq \lambda_{\kappa}^{i} \leq \operatorname{cf}(\kappa)^{+}$for $1 \leq i \leq 4$. If $\kappa$ is regular, then $\kappa<\mathfrak{b}_{\kappa}=\lambda_{\kappa}^{i}$ for $1 \leq i \leq 4$. In either case $\lambda_{\kappa}^{i}$ is regular for $1 \leq i \leq 4$

Recall the following result of W. Sierpiński and A. Zygmund [6]:

Proposition 5. $f \in \mathbb{R}^{\mathbb{R}}$ is in SZ if and only if $|[f=h]|<\mathfrak{c}$ for every continuous function $h$ defined on a $G_{\delta}$ set of cardinality $\mathfrak{c}$.

By Proposition 5 , if we let $J$ be the collection of functions $f \in \mathbb{R}^{\mathbb{R}}$ such that $\left.f\right|_{X}$ is continuous for some $G_{\delta}$-set $X \subseteq \mathbb{R}$ of cardinality $\mathfrak{c}$ and zero elsewhere, then $|J|=\mathfrak{c}$ and:

(*) $f \in \mathbb{R}^{\mathbb{R}} \in \mathrm{SZ}$ if and only if $|f \cap j|<\mathfrak{c}$ for every $j \in J$.

We can prove the following corollary of Theorems 3 which gives a partial answer to Problem 2. 
Corollary 6. $\lambda_{\mathfrak{c}}^{i}=\mathrm{C}_{\text {out }}(\mathrm{SZ})$ for all $1 \leq i \leq 4$.

Proof. Let $F \subseteq \mathcal{R}(\mathbb{R}, \mathbb{R})$ witness the definition of $\mathrm{C}_{\text {out }}(\mathrm{SZ})$, i.e., $|F|=$ $\mathrm{C}_{\text {out }}(\mathrm{SZ})$ and

$$
\left(\forall h \in \mathbb{R}^{\mathbb{R}}\right)(\exists f \in F)(h \circ f \notin \mathrm{SZ}) .
$$

By $(*)$ we know that $F$ has the following property,

$$
\left(\forall h \in \mathbb{R}^{\mathbb{R}}\right)(\exists f \in F)(\exists j \in J)(|[h \circ f=j]|=\mathfrak{c}) .
$$

It follows that $\lambda_{\mathfrak{c}}^{3} \leq \mathrm{C}_{\text {out }}(\mathrm{SZ})$. Letting $\kappa=\mathfrak{c}=|\mathbb{R}|$ Theorem 3 implies that $\lambda_{\mathfrak{c}}^{i} \leq \mathrm{C}_{\text {out }}(\mathrm{SZ})$ for all $1 \leq i \leq 4$.

We will be done if we show that $\mathrm{C}_{\text {out }}(\mathrm{SZ}) \leq \lambda_{\kappa}^{1}$. Let $\mathcal{N}$ denote the irrational numbers. Suppose $F \subseteq\left([\mathbb{R}]^{<\mathfrak{c}}\right)^{\mathbb{R}}$ and suppose $|F|<\mathrm{C}_{\text {out }}$ (SZ). Let $k: \mathcal{N} \rightarrow \mathbb{R}^{3}$ be a continuous bijection (see Exercise 7.15 of [4]).

For each $f \in F$ define the function $f_{1}$ on a subset of $\mathbb{R}^{3}$ by letting

$$
f_{1}(\langle x, y, z\rangle)=y
$$

for all $\langle x, y, z\rangle$ such that $x \in f(y)$ and $z \in f(y)$. Notice that $\left|f_{1}^{-1}(y)\right|<\mathfrak{c}$ for every $y \in \mathbb{R}$. We may extend $f_{1}$ to a $\mathcal{R}\left(\mathbb{R}^{3}, \mathbb{R}\right)$ type function $f_{1}^{*}$ defined on $\mathbb{R}^{3}$. Let $F^{*}=\left\{f_{1}^{*} \circ k: f \in F\right\}$. Clearly, $\left|F^{*}\right|<\mathrm{C}_{\text {out }}(\mathrm{SZ})$.

Let $\pi_{1}$ be the projection of $\mathbb{R}^{3}$ onto the first coordinate and $\pi_{3}$ be the projection of $\mathbb{R}^{3}$ onto the third coordinate. Since $\pi_{1} \circ k: \mathcal{N} \rightarrow \mathbb{R}$ is continuous and $F^{*} \subseteq \mathcal{R}(\mathcal{N}, \mathbb{R})$ has cardinality less than $\mathrm{C}_{\text {out }}(\mathrm{SZ})$, there is an $h: \mathbb{R} \rightarrow \mathbb{R}$ such that $\left|\left[h \circ\left(f_{1}^{*} \circ k\right)=\pi_{1} \circ k\right]\right|<\mathfrak{c}$ for every $f \in F$. Since $k$ is a bijection, we have $\left|\left[h \circ f_{1}^{*}=\pi_{1}\right]\right|<\mathfrak{c}$.

Fix an $f \in F$. We will be done if we show that $|\bigcup\{f(x): h(x) \in f(x)\}|<$ c. Let $y \in \bigcup\{f(x): h(x) \in f(x)\}$. There is a $x \in \mathbb{R}$ such that $y \in f(x)$ and $h(x) \in f(x)$. Now,

$$
\left(h \circ f_{1}^{*}\right)(\langle h(x), x, y\rangle)=h(x)=\pi_{1}(\langle h(x), x, y\rangle) .
$$

So, $y \in \pi_{3}\left[\left[\left(h \circ f_{1}^{*}\right)=\pi_{1}\right]\right]$. Since $\left|\left[\left(h \circ f_{1}^{*}\right)=\pi_{1}\right]\right|<\mathfrak{c}$, we have that $|\bigcup\{f(x): h(x) \in f(x)\}|<\mathfrak{c}$. Thus, $\mathrm{C}_{\text {out }}(\mathrm{SZ}) \leq \lambda_{\kappa}^{1}$.

Corollary 6 tells us that the continuous functions are as hard to avoid as any collection of $\mathfrak{c}$-many functions from $\mathbb{R}$ into $\mathbb{R}$, and that there is a single function that is as hard to avoid as the continuous functions. From Corollary 6 and Theorem 4 we get:

Corollary 7. If $\mathfrak{c}$ is regular, then $\mathrm{C}_{\text {out }}(\mathrm{SZ})=\mathfrak{b}_{\mathfrak{c}}>\mathfrak{c}$. If $\mathfrak{c}$ is singular, then $\operatorname{cf}(\mathfrak{c}) \leq \mathrm{C}_{\text {out }}(\mathrm{SZ}) \leq \mathrm{cf}(\mathfrak{c})^{+}$. In particular, $\mathrm{C}_{\text {out }}(\mathrm{SZ})$ is a regular cardinal.

Corollary 7 gives an affirmative answer to Problem 1 . Since $d_{\mathfrak{c}}>\mathfrak{c}$ [3], Corollary 7 , gives a negative answer to Problem 2 in the singular case. 
Question 1. Is it consistent that $\mathfrak{c}$ is regular and $\mathfrak{b}_{\mathfrak{c}}<d_{\mathfrak{c}}$ (see the second part Problem 2)?

Theorem 8. It is consistent that $\operatorname{cf}(\mathfrak{c})=\mathrm{C}_{\text {out }}(\mathrm{SZ})<\mathfrak{c}$.

Question 2. Is it consistent that $\mathfrak{c}$ is singular and $\mathrm{C}_{\text {out }}(\mathrm{SZ})=\operatorname{cf}(\mathfrak{c})^{+}$?

To prove Theorem 8 we will use the following theorem.

Theorem 9. $\mathfrak{b}_{\mathrm{cf}(\kappa)}<\kappa$ if and only if $\lambda_{\kappa}^{4}=\operatorname{cf}(\kappa)$.

Theorem 10. $\mathfrak{b}_{\kappa} \leq \mathfrak{b}_{\mathrm{cf}(\kappa)}$ for any cardinal $\kappa \geq \omega$. In particular, if $\kappa$ is a singular strong limit cardinal, then $\mathfrak{b}_{\kappa}<\kappa$.

An immediate corollary of Theorems 8, 9 and 10 is:

Corollary 11. It is consistent that $\mathfrak{b}_{\mathfrak{c}}<\mathfrak{c}$.

In light of Theorem 9 we could rephrase Question 2 to be: Is it consistent that $\mathfrak{c}$ is singular and $\mathfrak{c}<\mathfrak{b}_{\mathrm{cf}(\mathfrak{c})}$ ?

\section{Proofs of Theorems 3 and 4}

We first begin with some lemmas.

Lemma 12. $\lambda_{\kappa}^{1} \leq \lambda_{\kappa}^{4}$.

Proof. Let $F \subseteq \kappa^{\kappa}$ and suppose $|F|<\lambda_{\kappa}^{1}$. We will find an $h \in \kappa^{\kappa}$ such that $f[\{\xi: h(\xi) \leq f(\xi)\}]$ is bounded in $\kappa$ for every $f \in F$.

For each $f \in F$ define $f_{1} \in \mathcal{S}_{\kappa}$ so that $f_{1}(\xi)=\{\beta: \beta \leq f(\xi)+1\}$. Let $F_{1}=\left\{f_{1}: f \in F\right\}$. Since $\left|F_{1}\right| \leq|F|<\lambda_{\kappa}^{1}$ there is an $h \in \kappa^{\kappa}$ such that

$$
\bigcup\left\{f_{1}(\xi): h(\xi) \in f_{1}(\xi)\right\} \mid<\kappa
$$

for every $f_{1} \in F_{1}$. Since $f_{1}(\xi)$ is an initial segment of $\kappa$ for every $\xi \in \kappa$, (1) implies that $\bigcup\left\{f_{1}(\xi): h(\xi) \in f_{1}(\xi)\right\}$ is bounded in $\kappa$ by some $\alpha_{f_{1}} \in \kappa$ for every $f_{1} \in F_{1}$.

Fix $f \in F$. We claim that $f[\{\xi: h(\xi) \leq f(\xi)\}]$ is bounded above by $\alpha_{f_{1}} \in$ $\kappa$. Let $\beta \in f[\{\xi: h(\xi) \leq f(\xi)\}]$. Then, there is a $\gamma$ such that $h(\gamma) \leq f(\gamma)$ and $f(\gamma)=\beta$. So, $\beta=f(\gamma) \in f_{1}(\gamma) \subseteq \bigcup\left\{f_{1}(\xi): h(\xi) \in f_{1}(\xi)\right\} \subseteq \alpha_{f_{1}}$. Thus, $f[\{\xi: h(\xi) \leq f(\xi)\}]$ is bounded above by $\alpha_{f_{1}} \in \kappa$. Thus, $\lambda_{\kappa}^{1} \leq \lambda_{\kappa}^{4}$.

Lemma 13. $\lambda_{\kappa}^{3} \leq \lambda_{\kappa}^{2} \leq \lambda_{\kappa}^{1}$. 
Proof. It is obvious that $\lambda_{\kappa}^{3} \leq \lambda_{\kappa}^{2}$.

It should be noted that the proof of the inequality $\lambda_{\kappa}^{2} \leq \lambda_{\kappa}^{1}$ is very similar to the proof that $\mathrm{C}_{\text {out }}(\mathrm{SZ}) \leq \lambda_{\mathfrak{c}}^{1}$ in Corollary 6 . The differences between the two proofs stem from the fact that there is no topological structure to worry about in the proof that $\lambda_{\kappa}^{2} \leq \lambda_{\kappa}^{1}$.

Let $F \in\left[\mathcal{S}_{\kappa}\right]^{<\lambda_{\kappa}^{2}}$. We will find an $h \in \kappa^{\kappa}$ such that

$$
|\bigcup\{f(\xi): h(\xi) \in f(\xi)\}|<\kappa
$$

for every $f \in F$. Let $k: \kappa \rightarrow \kappa^{3}$ be a bijection.

For each $f \in F$ define the function $f_{1}$ on a subset of $\kappa^{3}$ by letting

$$
f_{1}(\langle x, y, z\rangle)=y
$$

for all $\langle x, y, z\rangle$ such that $x \in f(y)$ and $z \in f(y)$. Notice that $\left|f_{1}^{-1}(y)\right|<\kappa$ for every $y \in \kappa$. We may extend $f_{1}$ to a $\mathcal{R}\left(\kappa^{3}, \kappa\right)$ type function $f_{1}^{*}$ defined on $\kappa^{3}$.

Let $\pi_{1}$ be the projection of $\kappa^{3}$ onto the first coordinate and $\pi_{3}$ be the projection of $\kappa^{3}$ onto the third coordinate. Since $\left\{f_{1}^{*} \circ k: f \in F\right\} \subseteq \mathcal{R}(\kappa, \kappa)$ has cardinality less than $\lambda_{\kappa}^{2}$, there is an $h: \kappa \rightarrow \kappa$ such that $\mid\left[h \circ\left(f_{1}^{*} \circ k\right)=\right.$ $\left.\pi_{1} \circ k\right] \mid<\kappa$ for every $f \in F$. Since $k$ is a bijection, we have $\left|\left[h \circ f_{1}^{*}=\pi_{1}\right]\right|<\kappa$.

Fix an $f \in F$. We will be done if we show that $|\bigcup\{f(\xi): h(\xi) \in f(\xi)\}|<$ $\kappa$. Let $\gamma \in \bigcup\{f(\xi): h(\xi) \in f(\xi)\}$. There is a $\xi \in \kappa$ such that $\gamma \in f(\xi)$ and $h(\xi) \in f(\xi)$. Now,

$$
\left(h \circ f_{1}^{*}\right)(\langle h(\xi), \xi, \gamma\rangle)=h(\xi)=\pi_{1}(\langle h(\xi), \xi, \gamma\rangle) .
$$

So, $\gamma \in \pi_{3}\left[\left[h \circ f_{1}^{*}=\pi_{1}\right]\right]$. Now $|\bigcup\{f(\xi): h(\xi) \in f(\xi)\}|<\kappa$ since $\mid\left[h \circ f_{1}^{*}=\right.$ $\left.\pi_{1}\right] \mid<\kappa$.

Thus, $\lambda_{\kappa}^{2} \leq \lambda_{\kappa}^{1}$.

Lemma 14. $\lambda_{\kappa}^{4} \leq \mathfrak{b}_{\kappa}$.

Proof. Take an $F \subseteq \kappa^{\kappa}$ with $|F|<\lambda_{\kappa}^{4}$. For each $f \in F$ pick $f^{*} \in \kappa^{\kappa}$ so that

$$
f^{*}(\xi)=\max \{f(\xi), \xi\} .
$$

There is a $g \in \kappa^{\kappa}$ such that for every $f \in F$ we have $f^{*}\left[g \leq f^{*}\right]$ is bounded in $\kappa$. Since, by $(2), f^{*}$ maps unbounded sets to unbounded sets, we must have that $\left[g \leq f^{*}\right]$ is bounded in $\kappa$. Clearly, $[g \leq f] \subseteq\left[g \leq f^{*}\right]$. Thus, $|[g \leq f]|<\kappa$. Therefore, $\lambda_{\kappa}^{4} \leq \mathfrak{b}_{\kappa}$.

Lemma 15. If $\kappa$ is regular, then $\kappa<\mathfrak{b}_{\kappa}=\lambda_{\kappa}^{i}$ for $i \in\{1,2,3,4\}$. 
Proof. Lemmas 12, 13 and 14 yield that:

$$
\lambda_{\kappa}^{3} \leq \lambda_{\kappa}^{2} \leq \lambda_{\kappa}^{1} \leq \lambda_{\kappa}^{4} \leq \mathfrak{b}_{\kappa} .
$$

It remains to show that $\mathfrak{b}_{\kappa} \leq \lambda_{\kappa}^{3}$. Let $F \in[\mathcal{R}(\kappa, \kappa)]^{<\mathfrak{b}_{\kappa}}$. Fix $G \in\left[\kappa^{\kappa}\right]^{\kappa}$. We will construct an $h \in \kappa^{\kappa}$ such that $|[h \circ f=g]|<\kappa$ for every $g \in G$ and $f \in F$. Let $\left\{g_{\alpha}: \alpha \in \kappa\right\}$ be an enumeration of $G$.

For each $f \in F$ let $f^{*}(\alpha) \in \kappa$ be such that $\bigcup_{\xi \leq \alpha} f^{-1}(\xi) \subseteq f^{*}(\alpha)$ for each $\alpha \in \kappa$. Since $|F|<\mathfrak{b}_{\kappa}$ there is an $m \in \kappa^{\kappa}$ such that for every $f \in F$ we have $\left|\left[m \leq f^{*}\right]\right|<\kappa$.

Define $h \in \kappa^{\kappa}$ inductively so that for every $\alpha \in \kappa$ we select $h(\alpha)$ from the set

$$
\kappa \backslash\left\{g_{\beta}(x): x \in \bigcup\left\{\bigcup_{\xi \leq \alpha} f^{-1}(\xi): f \in F \& f^{*}(\alpha)<m(\alpha)\right\} \& \beta \leq \alpha\right\} .
$$

Note that such choices can be made since, for every $f$ such that $f^{*}(\alpha)<$ $m(\alpha)$ we have

$$
\bigcup_{\xi \leq \alpha} f^{-1}(\xi) \subseteq f^{*}(\alpha)<m(\alpha)
$$

So, $\bigcup\left\{\bigcup_{\xi \leq \alpha} f^{-1}(\xi): f \in F \& f^{*}(\alpha)<m(\alpha)\right\} \subseteq m(\alpha)<\kappa$.

We show that $h$ is as desired. Fix $f \in F$ and $g_{\beta} \in G$. For $\xi \in\left[h \circ f=g_{\beta}\right]$ we have

$$
h(f(\xi))=g_{\beta}(\xi) \in g_{\beta}\left[f^{-1}(f(\xi))\right] .
$$

By definition of $h$ we must have either $f(\xi)<\beta$ or $f^{*}(f(\xi)) \geq m(f(\xi))$. It follows that $\left|f\left[\left[h \circ f=g_{\beta}\right]\right]\right|<\kappa$. By regularity and the fact that $f \in \mathcal{R}(\kappa, \kappa)$, we have $\left|\left[h \circ f=g_{\beta}\right]\right|<\kappa$. Thus, $\mathfrak{b}_{\kappa} \leq \lambda_{\kappa}^{3}$.

Lemma 16. Let $F \in[\mathcal{R}(\kappa, \kappa)]^{<\lambda_{\kappa}^{4}}$. There is a bijection $k \in \kappa^{\kappa}$ such that for every $f \in F$ and $\alpha \in \kappa$

$$
\left|\bigcup_{\xi \leq \alpha} f^{-1}(k(\xi))\right|<\kappa
$$

Proof. For every $f \in F$ define $f^{*} \in \kappa^{\kappa}$ by $f^{*}(\alpha)=\left|f^{-1}(\alpha)\right|$. Let $F^{*}=$ $\left\{f^{*}: f \in F\right\}$. Since $\left|F^{*}\right| \leq|F|<\lambda_{\kappa}^{4}$, there is a $g \in \kappa^{\kappa}$ such that

$$
f^{*}\left[\left[g \leq f^{*}\right]\right]
$$

is bounded above by some $\rho_{f} \in \kappa$ for every $f \in F$. Notice that we may assume $g$ has unbounded range, since if we define $m \in \kappa^{\kappa}$ by $m(\xi)=$ $\max \{g(\xi), \xi\}$, then we will have $f^{*}\left[\left[m \leq f^{*}\right]\right] \subseteq f^{*}\left[\left[g \leq f^{*}\right]\right]$ for every $f \in F$. 
Define an injection $k^{*} \in \kappa^{\kappa}$ so that $\max \{\alpha, g(\alpha)\} \leq k^{*}(\alpha)$ for every $\alpha \in \kappa$. Since $\left|k^{*}[\kappa]\right|=\kappa$, there is an increasing onto function $h: k^{*}[\kappa] \rightarrow \kappa$. Let $k=\left(k^{*}\right)^{-1} \circ h^{-1}$.

We claim $k$ is as desired. Fix an $f \in F$. Let $\gamma \in \kappa$. Consider $\alpha \leq \gamma$. Since $h$ is increasing and $g$ has unbounded range, there is a $\beta_{\gamma} \in \kappa$ such that $h^{-1}(\alpha) \leq h^{-1}(\gamma) \leq g\left(\beta_{\gamma}\right)$. By definition of $k^{*}$, we have

$$
g\left(\beta_{\gamma}\right) \geq h^{-1}(\alpha)=k^{*}\left(\left(k^{*}\right)^{-1}\left(h^{-1}(\alpha)\right)\right)=k^{*}(k(\alpha)) \geq \max \{k(\alpha), g(k(\alpha))\} .
$$

So, for all $\alpha \leq \gamma$ we have $g(k(\alpha)) \leq g\left(\beta_{\gamma}\right)$.

Let $T$ be the set of all $\alpha \leq \gamma$ such that $f^{*}(k(\alpha))<g(k(\alpha))$ and $S=$ $(\gamma+1) \backslash T$. If $\alpha \in S$, then, by $(4), f^{*}(k(\alpha)) \leq \rho_{f}$. So, we have $\left|f^{-1}(k(\alpha))\right| \leq$ $\rho_{f}$ for $\alpha \in S$. If $\alpha \in T$, then

$$
\left|f^{-1}(k(\alpha))\right|=f^{*}(k(\alpha))<g(k(\alpha)) \leq g\left(\beta_{\gamma}\right) .
$$

Thus, $\left|\bigcup_{\alpha \leq \gamma} f^{-1}(k(\alpha))\right|$ is bounded above in $\kappa$ by $\max \left\{\rho_{f}, g\left(\beta_{\gamma}\right), \gamma\right\}$.

Lemma 17. If $\operatorname{cf}(\kappa)<\kappa$, then $\lambda_{\kappa}^{4} \leq \operatorname{cf}(\kappa)^{+}$.

Proof. Let $\left\{\Gamma_{\alpha}: \alpha \in \operatorname{cf}(\kappa)\right\}$ be a sequence of cardinals cofinal in $\kappa$. For every $\rho \in \operatorname{cf}(\kappa)^{+} \backslash \operatorname{cf}(\kappa)$ let $k_{\rho}: \rho \rightarrow \operatorname{cf}(\kappa)$ be a bijection. For each $\alpha \in \operatorname{cf}(\kappa)$ and $\rho \in \operatorname{cf}(\kappa)^{+} \backslash \operatorname{cf}(\kappa)$ let $f_{\alpha, \rho} \in \kappa^{\kappa}$ be defined by

$$
f_{\alpha, \rho}(\beta)= \begin{cases}\Gamma_{\alpha} & \text { if } \beta \notin \rho ; \\ \max \left\{\Gamma_{\alpha}, \Gamma_{k_{\rho}(\beta)}\right\} & \text { if } \beta \in \rho .\end{cases}
$$

Let $F=\left\{f_{\alpha, \rho}: \alpha \in \operatorname{cf}(\kappa) \& \rho \in \operatorname{cf}(\kappa)^{+} \backslash \operatorname{cf}(\kappa)\right\}$. Clearly, $|F|=\operatorname{cf}(\kappa)^{+}$.

Let $h \in \kappa^{\kappa}$ be arbitrary. We will find a $f_{\alpha, \rho} \in F$ such that $f_{\alpha, \rho}\left[\left[h \leq f_{\alpha, \rho}\right]\right]$ is unbounded in $\kappa$. First notice that

$$
\operatorname{cf}(\kappa)^{+}=\bigcup_{\alpha \in \operatorname{cf}(\kappa)}\left\{\beta \in \operatorname{cf}(\kappa)^{+}: h(\beta)<\Gamma_{\alpha}\right\} .
$$

Thus, there is an $\alpha \in \operatorname{cf}(\kappa)$ such that $\left|\left\{\beta \in \operatorname{cf}(\kappa)^{+}: h(\beta)<\Gamma_{\alpha}\right\}\right|=\operatorname{cf}(\kappa)^{+}$. Pick $W \subseteq\left\{\beta \in \operatorname{cf}(\kappa)^{+}: h(\beta)<\Gamma_{\alpha}\right\}$ such that $|W|=\operatorname{cf}(\kappa)$. There is a $\rho \in \operatorname{cf}(\kappa)^{+}$such that $W \subseteq \rho$. Now for each $w \in W$ we have $h(w) \leq$ $\Gamma_{\alpha} \leq f_{\alpha, \rho}(w)=\max \left\{\Gamma_{\alpha}, \Gamma_{k_{\rho}(w)}\right\}$. Since $|W|=\operatorname{cf}(\kappa),\left\{\Gamma_{k_{\rho}(w)}: w \in W\right\}$ is unbounded in $\kappa$. Thus, $f_{\alpha, \rho}\left[\left[h \leq f_{\alpha, \rho}\right]\right]$ is unbounded in $\kappa$.

Lemma 18. $\lambda_{\kappa}^{4} \leq \lambda_{\kappa}^{3}$. 
Proof. Notice the inequality follows from Lemma 15 in the case when $\kappa$ is regular. So, for the remainder of the proof we will assume that $\kappa$ is singular.

Let $F \in[\mathcal{R}(\kappa, \kappa)]^{<\lambda_{\kappa}^{4}}$. By Lemma 17 we have $|F|<\operatorname{cf}(\kappa)^{+}<\kappa$. Fix $G \in\left[\kappa^{\kappa}\right]^{\kappa}$. We construct an $h \in \kappa^{\kappa}$ such that $|[h \circ f=g]|<\kappa$ for every $g \in G$ and $f \in F$. Let $\left\{g_{\alpha}: \alpha \in \kappa\right\}$ be an enumeration of $G$.

By Lemma 16 there is a bijection $k \in \kappa^{\kappa}$ such that for each $f \in F$ and for every $\alpha \in \kappa$ we have

$$
\left|\bigcup_{\xi \leq \alpha} f^{-1}(k(\xi))\right|<\kappa
$$

For each $f \in F$ let $f^{*}(\alpha)=\left|\bigcup_{\xi \leq \alpha} f^{-1}(k(\xi))\right|$ for each $\alpha \in \kappa$. Since $|F|<\lambda_{\kappa}^{4}$ there is an $m \in \kappa^{\kappa}$ such that for every $f \in F$ the set $f^{*}\left[\left\{\xi: f^{*}(\xi) \geq m(\xi)\right\}\right]$ is bounded in $\kappa$.

Define $h \in \kappa^{\kappa}$ inductively so that for every $\alpha \in \kappa$ we select $h(k(\alpha))$ from the set

$\kappa \backslash\left\{g_{\beta}(x): x \in \bigcup\left\{\bigcup_{\xi \leq \alpha} f^{-1}(k(\xi)): f \in F \& f^{*}(\alpha)<m(\alpha)\right\} \& \beta \leq \alpha\right\}$.

Note that such choices can be made since,

$$
\left|\bigcup_{\xi \leq \alpha} f^{-1}(k(\xi))\right|=f^{*}(\alpha) \leq m(\alpha)
$$

for every $f \in F$ such that $f^{*}(\alpha)<m(\alpha)$. So,

$$
\bigcup\left\{\bigcup_{\xi \leq \alpha} f^{-1}(k(\xi)): f \in F \& f^{*}(\alpha)<m(\alpha)\right\} \mid \leq \max \{|F|, m(\alpha)\}<\kappa .
$$

We show that $h$ is as desired. Fix $f \in F$ and $g_{\beta} \in G$. For $\xi \in\left[h \circ f=g_{\beta}\right]$ pick $\alpha_{\xi}$ so that $k\left(\alpha_{\xi}\right)=f(\xi)$. We now have

$$
h\left(k\left(\alpha_{\xi}\right)\right)=h(f(\xi))=g_{\beta}(\xi) \in g_{\beta}\left[f^{-1}\left(k\left(\alpha_{\xi}\right)\right)\right] .
$$

By definition of $h$ we must have $\alpha_{\xi}<\beta$ or $f^{*}\left(\alpha_{\xi}\right) \geq m\left(\alpha_{\xi}\right)$. Let $T=$ $\left\{\alpha_{\xi}: f^{*}\left(\alpha_{\xi}\right) \geq m\left(\alpha_{\xi}\right)\right\}$.

By our choice of $m$, the set $f^{*}[T]$ is bounded in $\kappa$ by some cardinal $\theta$. Since $\kappa=\bigcup_{\alpha \in \kappa} \bigcup_{\xi \leq \alpha} f^{-1}(k(\xi))$ and $\kappa$ is singular, there is a $\pi \in \kappa$ such that $f^{*}(\zeta)=\left|\bigcup_{\xi \leq \zeta} f^{-1}(k(\xi))\right|>\theta$ for all $\zeta>\pi$. Thus, $T$ is bounded by $\pi$.

We now have that $M=\left\{\alpha_{\xi}: \xi \in\left[h \circ f=g_{\beta}\right]\right\}$ is bounded in $\kappa$ by $\max \{\beta, \sup (T)\}$. Since $M$ is bounded, we have, by (5), $\left|f^{-1}(k[M])\right|<\kappa$. 

So,

Let $\xi \in\left[h \circ f=g_{\beta}\right]$. Then there is an $\alpha_{\xi} \in M$ such that $f(\xi)=k\left(\alpha_{\xi}\right)$.

$$
\xi \in f^{-1}(f(\xi))=f^{-1}\left(k\left(\alpha_{\xi}\right)\right) \subseteq f^{-1}(k[M]) .
$$

Thus, $\left[h \circ f=g_{\beta}\right] \subseteq f^{-1}(k[M])$. Since $\left.\mid f^{-1}(k[M])\right] \mid<\kappa$, we must have $\left|\left[h \circ f=g_{\beta}\right]\right|<\kappa$. Thus, $\lambda_{\kappa}^{4} \leq \lambda_{\kappa}^{3}$.

Proof of Theorem 3. It is obvious that $\mathrm{cf}(\kappa) \leq \lambda_{\kappa}^{4}$.

Lemmas 12, 18, and 13 yield that $\lambda_{\kappa}^{i}=\lambda_{\kappa}^{1}$ for all $1 \leq i \leq 4$.

Proof of Theorem 4. When $\kappa$ is singular Lemma 17 and Theorem 3 yield that $\operatorname{cf}(\kappa) \leq \lambda_{\kappa}^{i} \leq \operatorname{cf}(\kappa)^{+}$for $1 \leq i \leq 4$.

Suppose now that $\kappa$ is regular. By Lemma $15, \kappa<\mathfrak{b}_{\kappa}=\lambda_{\kappa}^{i}$ for all $1 \leq i \leq 4$.

\section{Proofs of Theorems 10 and 9}

It will be useful to define some other cardinal numbers which will be shown to be equal to the bounding number. Put

$$
\begin{aligned}
\mathfrak{b}(\kappa, \operatorname{cf}(\kappa))=\min \left\{|F|: F \subseteq \operatorname{cf}(\kappa)^{\kappa}\right. & \\
& \left.\&\left(\forall g \in \operatorname{cf}(\kappa)^{\kappa}\right)(\exists f \in F)(|[g \leq f]|=\kappa)\right\}, \\
\mathfrak{b}^{b d}(\kappa, \operatorname{cf}(\kappa))=\min \left\{|F|: F \subseteq \operatorname{cf}(\kappa)^{\kappa}\right. & \left.\quad \&\left(\forall g \in \operatorname{cf}(\kappa)^{\kappa}\right)(\exists f \in F)([g \leq f] \text { is unbounded in } \kappa)\right\} .
\end{aligned}
$$

Lemma 19. $\mathfrak{b}_{\kappa}=\mathfrak{b}(\kappa, \operatorname{cf}(\kappa))=\mathfrak{b}^{b d}(\kappa, \operatorname{cf}(\kappa))$.

Proof. The lemma is obvious if $\kappa$ is regular so we assume that $\kappa$ is singular. Let $P=\left\{\lambda_{\alpha}: \alpha \in \operatorname{cf}(\kappa)\right\}$ be an increasing cofinal sequence of regular cardinals in $\kappa$ such that $\lambda_{0}>\operatorname{cf}(\kappa)$.

We first claim that $\mathfrak{b}_{\kappa} \leq \mathfrak{b}(\kappa, \operatorname{cf}(\kappa))$. Let $F \subseteq \operatorname{cf}(\kappa)^{\kappa}$ witness the definition of $\mathfrak{b}(\kappa, \operatorname{cf}(\kappa))$. For each $f \in F$ define $f^{*} \in \kappa^{\kappa}$ by $f^{*}(\beta)=\lambda_{f(\beta)}$. Let $F^{*}=\left\{f^{*}: f \in F\right\}$ and note that $\left|F^{*}\right| \leq \mathfrak{b}(\kappa, \operatorname{cf}(\kappa))$. Pick $g \in \kappa^{\kappa}$ and define $g^{1} \in \operatorname{cf}(\kappa)^{\kappa}$ by $g^{1}(\beta)=\min \left\{\alpha \in \operatorname{cf}(\kappa): g(\beta) \leq \lambda_{\alpha}\right\}$. There is, by definition of $\mathfrak{b}(\kappa, \operatorname{cf}(\kappa))$, an $f \in F$ such that $\left|\left[g^{1} \leq f\right]\right|=\kappa$. If $\beta \in\left[g^{1} \leq f\right]$, then $g(\beta) \leq \lambda_{g^{1}(\beta)} \leq \lambda_{f(\beta)}=f^{*}(\beta)$. So, $\left|\left[g \leq f^{*}\right]\right|=\kappa$. Thus, $\mathfrak{b}_{\kappa} \leq \mathfrak{b}(\kappa, \operatorname{cf}(\kappa))$.

We now claim that $\mathfrak{b}(\kappa, \operatorname{cf}(\kappa)) \leq \mathfrak{b}^{b d}(\kappa, \operatorname{cf}(\kappa))$. Let $H: \kappa \rightarrow P$ be defined by, $H(\beta)=\min \left\{\lambda_{\alpha}: \beta \leq \lambda_{\alpha}\right\}$. Let $S=\{\langle\beta, \zeta\rangle \in \kappa \times \kappa: \zeta \leq H(\beta)\}$, note that $|S|=\kappa$. For each $\beta \in \kappa$ let $S_{\beta}=\{\langle\xi, \zeta\rangle \in S: \xi=\beta$. Let $F \subseteq \operatorname{cf}(\kappa)^{\kappa}$ witness the definition of $\mathfrak{b}^{b d}(\kappa, \operatorname{cf}(\kappa))$. For each $f \in F$ let $f^{*}: S \rightarrow \operatorname{cf}(\kappa)$ be defined so that $f^{*}\left[S_{\beta}\right]=\{f(\beta)\}$ for every $\beta \in \kappa$. Let 
$F^{*}=\left\{f^{*}: f \in F\right\}$, note $\left|F^{*}\right| \leq|F|$. Pick $g \in \operatorname{cf}(\kappa)^{S}$. For every $\beta \in \kappa$ we have that $\left|S_{\beta}\right|$ is regular and strictly larger than $\operatorname{cf}(\kappa)$, so there is an $\zeta_{\beta} \in \operatorname{cf}(\kappa)$ such that $\left|S_{\beta} \cap g^{-1}\left(\zeta_{\beta}\right)\right|=\left|S_{\beta}\right|$. Define $g^{1} \in \operatorname{cf}(\kappa)^{\kappa}$ so that $g^{1}(\beta)=\zeta_{\beta}$ for every $\beta \in \kappa$. There is, by definition of $\mathfrak{b}^{b d}(\kappa, \operatorname{cf}(\kappa))$, an $f \in F$ such that $\left[g^{1} \leq f\right]$ is unbounded in $\kappa$. We will be done if we show that $\left|\left[g \leq f^{*}\right]\right|=\kappa$. Let $\beta \in\left[g^{1} \leq f\right]$. For $x \in S_{\beta} \cap g^{-1}\left(\zeta_{\beta}\right)$ we have $g(x)=\zeta_{\beta}=g^{1}(\beta) \leq f(\beta)=f^{*}(x)$. So, $S_{\beta} \cap g^{-1}\left(\zeta_{\beta}\right) \subseteq\left[g \leq f^{*}\right]$ for $\beta \in\left[g^{1} \leq f\right]$. Since $\left[g^{1} \leq f\right]$ is unbounded and $\left|S_{\beta} \cap g^{-1}\left(\zeta_{\beta}\right)\right|=\left|S_{\beta}\right| \geq \beta$ we have $\left|\left[g \leq f^{*}\right]\right|=\kappa$. Thus, $\mathfrak{b}(\kappa, \operatorname{cf}(\kappa)) \leq \mathfrak{b}^{b d}(\kappa, \operatorname{cf}(\kappa))$.

We show that $\mathfrak{b}^{b d}(\kappa, \operatorname{cf}(\kappa)) \leq \mathfrak{b}_{\kappa}$. Let $F \subseteq \kappa^{\kappa}$ witness the definition of $\mathfrak{b}_{\kappa}$. For each $f \in F$ let $f^{*} \in P^{\kappa}$ be defined by $f^{*}(\xi)=\min \{\alpha \in \operatorname{cf}(\kappa): f(\xi) \leq$ $\left.\lambda_{\alpha}\right\}$. Let $F^{*}=\left\{f^{*}: f \in F\right\}$. Clearly, $\left|F^{*}\right| \leq|F|$. It is easy to check that $\left|F^{*}\right|$ satisfies the condition in the definition of $\mathfrak{b}^{b d}(\kappa, \operatorname{cf}(\kappa))$. Thus, $\mathfrak{b}^{b d}(\kappa, \operatorname{cf}(\kappa)) \leq \mathfrak{b}_{\kappa}$.

Proof of Theorem 10. Let $P$ be as in the proof of Lemma 19. By Lemma 19, it is enough to show that $\mathfrak{b}^{b d}(\kappa, \operatorname{cf}(\kappa)) \leq \mathfrak{b}_{\mathrm{cf}(\kappa)}$. Let $F \subseteq \operatorname{cf}(\kappa)^{P}$ witness the definition of $\mathfrak{b}_{\mathrm{cf}(\kappa)}$. For each $f \in F$ define $f^{*} \in \operatorname{cf}(\kappa)^{\kappa}$ so that $\left.f^{*}\right|_{P}=\left.f\right|_{P}$ and arbitrarily elsewhere. Let $F^{*}=\left\{f^{*}: f \in F\right\}$, note $\left|F^{*}\right| \leq$ $|F|$. Let $g \in \operatorname{cf}(\kappa)^{\kappa}$. We may find an $f \in F$ such that $\left|\left[\left.g\right|_{P} \leq\left. f\right|_{P}\right]\right|=\operatorname{cf}(\kappa)$. It follows that $\left[g \leq f^{*}\right]$ is unbounded in $\kappa$. Thus, $\mathfrak{b}^{b d}(\kappa, \operatorname{cf}(\kappa)) \leq \mathfrak{b}_{\operatorname{cf}(\kappa)}$ Therefore, $\mathfrak{b}_{\kappa} \leq \mathfrak{b}_{\mathrm{cf}(\kappa)}$.

If $\kappa$ is a strong singular limit cardinal, then $\mathfrak{b}_{\kappa} \leq \mathfrak{b}_{\operatorname{cf}(\kappa)} \leq 2^{\mathrm{cf}(\kappa)}<\kappa$.

Proof of Theorem 9. By Theorem 4, both conditions in the equivalence we are trying to prove are false when $\kappa$ is regular. So, we may assume that $\kappa$ is singular. We also may assume that $\kappa \neq \mathfrak{b}_{\mathrm{cf}(\kappa)}$ since $\mathfrak{b}_{\lambda}$ is regular for any infinite cardinal $\lambda$.

Let $\left\{\lambda_{\alpha}: \alpha \in \operatorname{cf}(\kappa)\right\}$ be an increasing cofinal sequence of regular cardinals in $\kappa$.

Suppose $\kappa<\mathfrak{b}_{\operatorname{cf}(\kappa)}$. Let $F \subseteq \kappa^{\kappa}$ and $|F|=\operatorname{cf}(\kappa)$. Let $\left\{f_{\xi}: \xi \in \operatorname{cf}(\kappa)\right\}$ be an enumeration of $F$. We will find a $h \in \kappa^{\kappa}$ such that $f[[h \leq f]]$ is bounded in $\kappa$ for every $f \in F$. For each $\alpha \in \kappa$ let $g_{\alpha} \in \operatorname{cf}(\kappa)^{\operatorname{cf}(\kappa)}$ be defined so that $\lambda_{g_{\alpha}(\xi)}>f_{\xi}(\alpha)$. Since $\kappa<\mathfrak{b}_{\mathrm{cf}(\kappa)}$, there is a $j \in \operatorname{cf}(\kappa)^{\mathrm{cf}(\kappa)}$ such that $\left|\left[j \leq g_{\alpha}\right]\right|<\operatorname{cf}(\kappa)$ for every $\alpha \in \kappa$. For each $\alpha \in \kappa$ let $\beta_{\alpha} \in \operatorname{cf}(\kappa)$ be such that

$$
\beta_{\alpha}>\sup \left\{g_{\alpha}(\xi): g_{\alpha}(\xi) \geq j(\xi)\right\} .
$$

Define $h \in \kappa^{\kappa}$ so that $h(\alpha)=\lambda_{\beta_{\alpha}}$.

We show that $f[[h \leq f]]$ is bounded in $\kappa$ for every $f \in F$. Fix $\xi \in \operatorname{cf}(\kappa)$. Suppose $h(\alpha) \leq f_{\xi}(\alpha)$. We now have $\lambda_{\beta_{\alpha}} \leq f_{\xi}(\alpha)<\lambda_{g_{\alpha}(\xi)}$. It follows 
that $\beta_{\alpha}<g_{\alpha}(\xi)$. Thus, by $(6), g_{\alpha}(\xi)<j(\xi)$. So, $f_{\xi}(\alpha)<\lambda_{g_{\alpha}(\xi)}<\lambda_{j(\xi)}$. Thus, $f_{\xi}\left[\left[h \leq f_{\xi}\right]\right]$ is bounded in $\kappa$ by $\lambda_{j(\xi)}$. Therefore, $\lambda_{\kappa}^{4}>\operatorname{cf}(\kappa)$ and by Theorem 4 we get $\lambda_{\kappa}^{4}=\operatorname{cf}(\kappa)^{+}$.

Now suppose $\mathfrak{b}_{\operatorname{cf}(\kappa)}<\kappa$. Let $\Gamma^{*} \subseteq \operatorname{cf}(\kappa)^{\operatorname{cf}(\kappa)}$ be such that $\left|\Gamma^{*}\right|=\mathfrak{b}_{\mathrm{cf}(\kappa)}$ and for every $f \in \operatorname{cf}(\kappa)^{\operatorname{cf}(\kappa)}$ there is a $\gamma^{*} \in \Gamma^{*}$ such that $\left|\left[f \leq \gamma^{*}\right]\right|=\operatorname{cf}(\kappa)$. Let $\left\{\gamma_{\xi}^{*}: \xi \in \mathfrak{b}_{\operatorname{cf}(\kappa)}\right\}$ be a well ordering of $\Gamma^{*}$. Inductively, define $\Gamma=\left\{\gamma_{\xi} \in\right.$ $\left.\operatorname{cf}(\kappa)^{\operatorname{cf}(\kappa)}: \xi \in \mathfrak{b}_{\operatorname{cf}(\kappa)}\right\}$ so that for every $\xi \in \mathfrak{b}_{\mathrm{cf}(\kappa)}$ we have $\left|\left[\gamma_{\xi} \leq \gamma_{\alpha}^{*}\right]\right|<\operatorname{cf}(\kappa)$ and $\left|\left[\gamma_{\xi} \leq \gamma_{\alpha}\right]\right|<\operatorname{cf}(\kappa)$ for every $\alpha<\xi$. Notice that $\Gamma$ has the property that

$$
\begin{aligned}
\left(\forall M \in[\Gamma]^{\mathfrak{b}_{\operatorname{cf}(\kappa)}}\right)\left(\forall f \in \operatorname{cf}(\kappa)^{\operatorname{cf}(\kappa)}\right) & (\exists m \in M) \\
& (|[f \leq m]|=\operatorname{cf}(\kappa)) .
\end{aligned}
$$

For each $\alpha \in \operatorname{cf}(\kappa)$ let $f_{\alpha}: \Gamma \rightarrow \kappa$ be defined by $f_{\alpha}(\gamma)=\lambda_{\gamma(\alpha)}$. Let $F=\left\{f_{\alpha}: \alpha \in \operatorname{cf}(\kappa)\right\}$.

Since $|\Gamma|=\mathfrak{b}_{\operatorname{cf}(\kappa)}<\kappa$, we may identify $\Gamma$ with a subset of $\kappa$. We claim that for every $A \in[\Gamma]^{\mathfrak{b}_{\mathrm{cf}}(\kappa)}$ there is an $f \in F$ such that $f[A]$ is unbounded in $\kappa$. By way of contradiction assume that there is an $A \in[\Gamma]^{\mathfrak{b}_{\mathrm{cf}(\kappa)}}$ such that $f_{\alpha}[A]$ is bounded in $\kappa$ for every $\alpha \in \operatorname{cf}(\kappa)$. Then for every $\alpha \in \operatorname{cf}(\kappa)$ there exists a $\beta_{\alpha} \in \mathrm{cf}(\kappa)$ such that $\lambda_{\gamma(\alpha)}=f_{\alpha}(\gamma)<\lambda_{\beta_{\alpha}}$ for every $\gamma \in A$. In particular, we have that $\gamma(\alpha)<\beta_{\alpha}$ for every $\gamma \in A$ and every $\alpha \in \operatorname{cf}(\kappa)$, contradicting (7). So, the claim is proved.

For every $f \in F$ define $f^{*} \in \kappa^{\kappa}$ so that $f^{*}(\beta)=f(\beta)$ if $\beta \in \Gamma$ and $f^{*}(\beta)=0$ if $\beta \notin \Gamma$. Let $F^{*}=\left\{f^{*}: f \in F\right\}$. Since $\left|F^{*}\right|=\operatorname{cf}(\kappa)$, we will be done if we show that for every $g \in \kappa^{\kappa}$ there is an $f^{*} \in F^{*}$ such that $f^{*}\left[g \leq f^{*}\right]$ is unbounded in $\kappa$. Let $g \in \kappa^{\kappa}$. Since $|\Gamma|=\mathfrak{b}_{\operatorname{cf}(\kappa)}$ is regular and $\operatorname{cf}(\kappa)<|\Gamma|$ there is an $A \in[\Gamma]^{\mathfrak{b}_{\mathrm{cf}(\kappa)}}$ such that $g[A]$ is bounded in $\kappa$. By the claim from the previous paragraph, there is an $f \in F$ such that $f[A]$ is unbounded in $\kappa$. It follows that $f\left[\left[\left.g\right|_{\Gamma} \leq f\right]\right]$ is unbounded in $\kappa$. Thus, $f^{*}\left[g \leq f^{*}\right]$ is unbounded in $\kappa$. Therefore, $\lambda_{\kappa}^{4} \leq \operatorname{cf}(\kappa)$.

The inequality $\operatorname{cf}(\kappa) \leq \lambda_{\kappa}^{4}$ follows from Theorem 3 . Thus, $\operatorname{cf}(\kappa)=\lambda_{\kappa}^{4}$.

\section{Proof of Theorem 8}

By Theorem 9 and Corollary 6, it is enough to find a model of ZFC such that $\mathfrak{b}_{\mathrm{cf}(\mathfrak{c})}<\mathfrak{c}$.

The argument we present is very standard, but we include it for those who are not very familiar with forcing.

A summary of the argument is that we add $\omega_{\omega_{1}}$ Cohen reals to a model of ZFC+GCH. In the resulting universe $\mathfrak{c}=\omega_{\omega_{1}}$ and the functions of the ground model witness that $\mathfrak{b}_{\omega_{1}}=\omega_{2}$ (this works because the forcing is ccc). 
We now give the detail version of the argument. Let $M$ be a countable transitive model of $\mathrm{ZFC}+\mathrm{GCH}$. Let $\mathcal{P} \in M$ be the poset of partial functions with finite domains from $\omega_{\omega_{1}}^{M}$ into 2 ordered by reverse inclusion. Let $G$ be an $M$ generic filter in $\mathcal{P}$. We claim that $\left(\mathfrak{b}_{\mathrm{cf}(\mathfrak{c})}<\mathfrak{c}\right)^{M[G]}$. Since $\mathcal{P}$ satisfies the countable chain condition, by [2, Theorem 9.2.11], we have $\omega_{1}^{M}=\omega_{1}^{M[G]}$. Since we have GCH in $M$, we have $\left(\left|\omega_{1}^{\omega_{1}}\right|=\omega_{2}\right)^{M}$. So there is a bijection $h: \omega_{1}^{\omega_{1}} \rightarrow \omega_{2}$ in $M$. Since being a bijection is an absolute property we have that $h: M \cap\left(\omega_{1}^{\omega_{1}}\right) \rightarrow \omega_{2}$ is a bijection in $M[G]$. Thus, $\left(\left|M \cap \omega_{1}^{\omega_{1}}\right|=\omega_{2}\right)^{M[G]}$. We claim that $M \cap \omega_{1}^{\omega_{1}}$ witnesses the definition

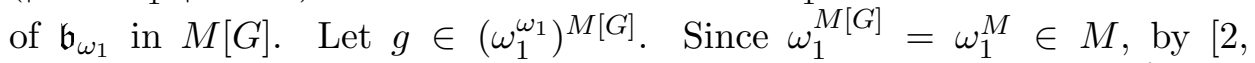
Corollary 9.2.9], there is a function $h$ in $M$ such that $h: \omega_{1} \rightarrow\left[\omega_{1}\right] \leq \omega$ and $g(x) \in h(x)$ for every $x \in \omega_{1}$. Define in $M$ the function $h^{*}: \omega_{1} \rightarrow \omega_{1}$ by $h^{*}(x)=\sup (h(x))+1$. Now $h^{*} \in M \cap \omega_{1}^{\omega_{1}}$ and $g<h^{*}$. So the claim is established. Thus, $\left(\mathfrak{b}_{\omega_{1}}=\omega_{2}\right)^{M[G]}$. Since GCH holds in $M$, it can be shown that $\left|\left(\omega_{\omega_{1}}\right)^{\omega}\right|=\omega_{\omega_{1}}$ in $M$. So by [2, Corollary 9.4.9] we have $\left(\mathfrak{c}=\omega_{\omega_{1}}\right)^{M[G]}$. Thus, $\left(\operatorname{cf}(\mathfrak{c})=\omega_{1}\right)^{M[G]}$. So, $\left(\mathfrak{b}_{\mathrm{cf}(\mathfrak{c})}<\mathfrak{c}\right)^{M[G]}$.

\section{References}

[1] Bartoszyński, T. and Judah, H., Set Theory: On the Structure of the Real Line, A. K. Peters, Wellesley, 1995.

[2] Ciesielski, K., Set Theory for the Working Mathematician, London Math. Soc. Stud. Texts 39, Cambridge Univ. Press, Cambridge, 1997.

[3] Ciesielski, K. and Natkaniec, T., Algebraic properties of the class of SierpinskiZygmund functions, Topology Appl. 79 (1997), 75-99.

[4] Kechris, A., Classical Descriptive Set Theory, Grad. Texts in Math. 156, Springer, New York, 1995.

[5] Shelah, S., On Cielsielski's problems, J. Appl. Anal. 3(2) (1997), 191-209.

[6] Sierpiński, W. and Zygmund, A., Sur une fonction qui est discontinue sur tout ensemble de puissance du continu, Fund. Math. 4 (1923), 316-318.

FRANCIS JORDAN

Department of Mathematics

LOYOLA UNIVERSITY

New Orleans, LA 70118

USA

EMAIL: FEJORD@HOTMAIL.COM 